Novel nematode-derived insecticidal proteins for pest control

Dillman, A.R. University of California, Riverside CA

Ment, D. Agricultural Research Organization

Project award year: 2018

One year feasibility project 


\section{Novel nematode-derived insecticidal proteins for pest control.}

Objectives. The goal of our 1-year feasibility study was to identify and characterize the insecticidal proteins released by the insect-parasitic nematode Steinernema feltiae and to determine the feasibility of using these proteins as feed bio-toxins against herbivorous pests from two different groups of insects; beetles and larval moths and butterflies. During the course of the project we decided to also evaluate the potential of the proteins released by St. carpocapsae, a parasitic nematode closely related to St.feltiae.

Background. Food security and efficient agricultural production are two of the most important global challenges of our era. Insect pests pose are major problem in agricultural systems, causing crop losses between 36\%-42\% in some staple crop systems. Reducing insect damage is required to increase the efficiency of food production and food security. Toxins from the insect pathogen Bacillus thuringiensis $(\mathrm{Bt})$ are widely used in biological control. Bt toxins are specifically toxic to insects and are applied or expressed by transgenic crops in pest management in agriculture and forestry. However, there is growing concern due to development of resistance, which may lead to ineffectiveness of Bt toxins. One way to cope with the challenge of growing resistance to Bt toxins is to replace them, or supplement them with other bio-toxins. We have recently discovered that a specialized group of insect-parasitic nematodes produce and release insecticidal proteins while interacting with its hosts (Lu et al., 2017). As part of this funded feasibility study, we began to characterize these novel bio-toxins and to evaluate their toxicology toward insect hosts.

Conclusions, solutions, and achievements. We have fractionated the crude proteins released by Steinenernema feltiae nematodes when they are activated by exposure to Galleria mellonella tissue. We had previously determined that $S$. feltiae releases a mixture of $\sim 250$ different proteins when activated. Using bioassay-guided fractionation, we found that only a few fractions retained high toxicity. We performed mass spectrometry on the toxic fractions as well as the crude ESPs and have identified 6 candidate proteins with high significance that are enriched $\geq 20$ fold in the toxic fractions. We also performed oral toxicity assays using crude ESPs collected from St.feltiae and St. carpocasae against several different insect hosts, including Spodoptera littoralis moth larvae and Alphitobius diaperinus beetle larvae. Both of these insects are considered agricultural pests. We found that the ESPs of St. carpocapsae have high oral toxicity to both the larvae of Sp. littoralis and $A$. diaperinus. We also have promising preliminary data suggesting that the crude proteins released by St. feltiae have oral toxicity to Sp. littoralis, though these experiments have not been completed just yet. We are highly encouraged by the finding of strong oral toxicity against Sp. littoralis and A.diaperinus and the identification of candidate toxin genes. Future experiments include testing individual recombinant proteins for insecticidal activity and producing transgenic plants expressing individual candidate toxin proteins that have been validated for toxicity both by injection and oral ingestion. This project could lead to the identification and characterization of new insecticidal proteins.

Implications. Our findings suggest that the toxins produced by insect-parasitic nematodes could be useful in the control of pest insects. These toxins may represent an entirely new class of previously unexamined insecticides and merit additional study. 
Contribution of the colaboration. This project was divided into two different components; harvesting and analyzing nematode excreted/secreted proteins (ESPs) and performing oral toxicity assays with agricultural pests. The Dillman lab (US PI) was in charge of producing the nematode ESPs for both St. carpocapsae and St. feltiae and performing the subsequent fractionation and analysis of the proteins contained in the ESPs. They first assessed toxicity of the crude ESPs by direct injection of 20 nanograms into adult fruit flies. They then performed the subsequent fractionation and worked to identify individual candidate toxins. The Ment lab (Israel PI) was in charge of leading the oral toxicity assays and developing different ways of assessing the potential usefulness of the nematode ESPs in biological control. They performed feeding assays using larvae of Sp. littoralis and A. diaperinus. Both labs exchanged written communication and participated in virtual meetings to discuss results and plan additional experiments. This division of labor leveraged the expertise of both labs and facilitated the performance of experiments that neither lab alone was capable of doing. 
Achievements. We were able to successfully induce parasitic activation of St.feltiae and St. carpocapsae infective juveniles to produce and release crude excreted/secreted proteins (ESPs). Using anion exchange fractionation and fast protein liquid chromatography we were able to separate the $\sim 250$ proteins present in the crude ESPs of St.feltiae into $\sim 70$ different fractions (Fig. 1). We injected 10 nanograms of these protein fractions into adult fruit flies and found that only $\sim 8$ of the fractions had toxic activity. We then identified all of the proteins present in the crude ESP mixture as well as one of the toxic fractions. By comparing the resulting data, we identified 6 proteins that are highly enriched in the toxic fraction ( $\geq 20$ fold enriched). These 6 proteins are the candidate toxins that we wish to study moving forward.

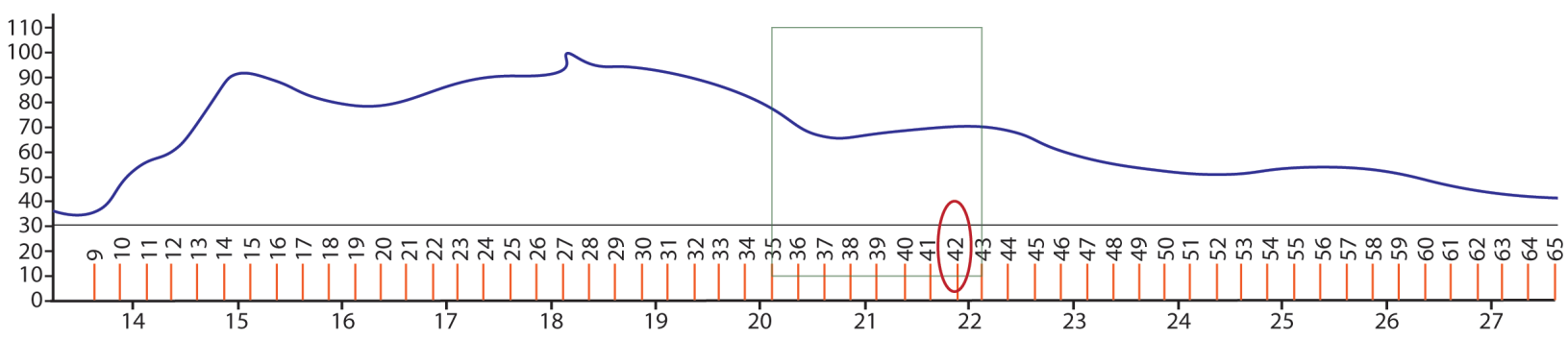

Figure 1. Only some fractions of $S$. feltiae ESPs have insecticidal activity. S. feltiae ESPs were separated into many fractions using an anion exchange column. The $\mathrm{Y}$ axis shows relative amounts of protein eluted using FPLC, over time ( $\mathrm{X}$ axis). The blue line shows the overall amount of protein eluting over time. Five nanograms of every other fraction were injected into fruit flies. Fractions 35-42, highlighted by a green box, showed some level of toxicity. Fraction 42 was used for mass spectrometry to identify candidate toxins.

In order to determine the feasibility of using the nematode-derived toxins in agriculture as a means of biological control of insect pests, we evaluated the toxicity of crude ESPs from St.feltiae and St. carpocapsae by feeding them to the larvae of Sp. littoralis and A. diaperinus. We found that around $50 \%$ of neonatal Sp. littoralis were paralyzed after 10-15 hours after they began feeding on castor leaf discs impregnated with St. carpocapsae crude toxins (8.5 $\mu \mathrm{g} / \mathrm{per}$ leaf disc). After 48 hours, $80-100 \%$ mortality was observed, whereas we observed no mortality at 48 hours for control experiments (Fig. 2). We also observed promising toxicity of St. feltiae toxins being fed to Sp. littoralis neonates. We have also found that St. carpocapsae crude toxins have toxicity against $A$. diaperinus first- and second-instar beetle larvae. These are the major achievements of our oneyear feasibility study. Additional experiments need to be performed before the results will be submitted for publication, however, we plan to utilize these results in a full BARD proposal later this year. 


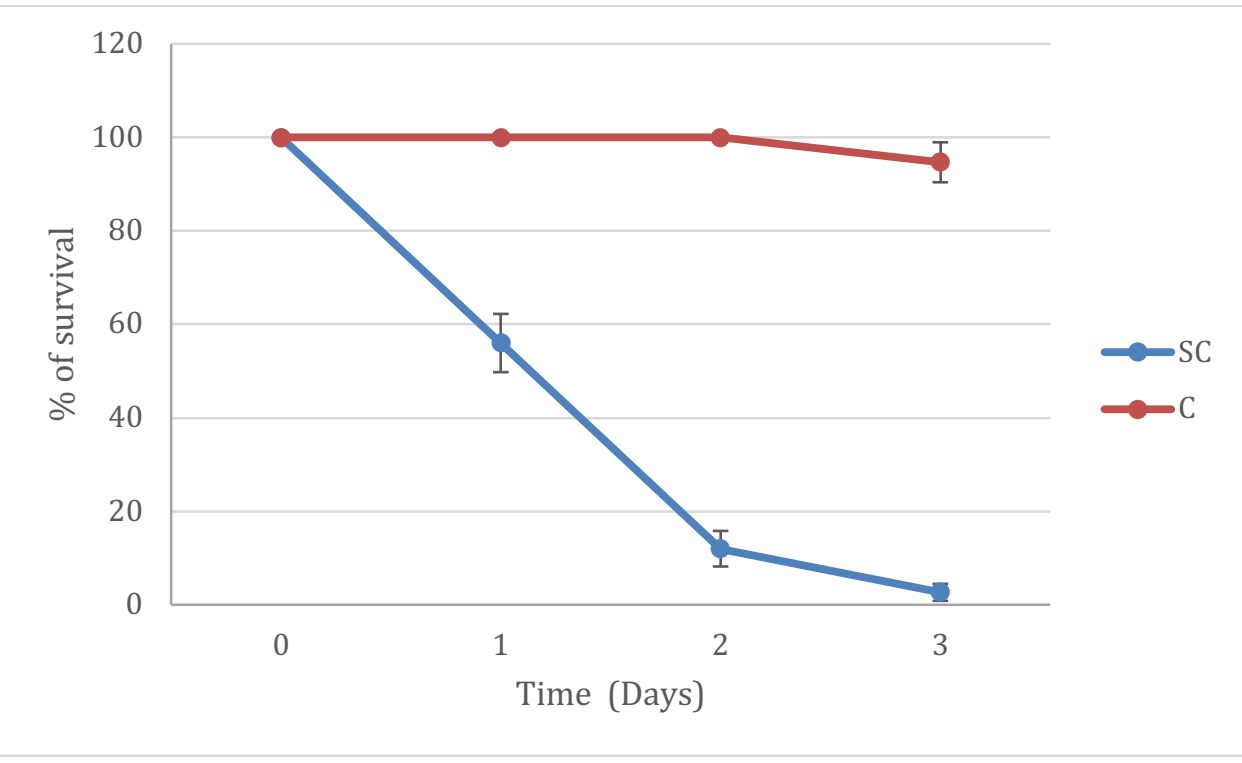

Figure 2.Sp. littoralis oral toxicity feeding assay. St. carpocapsae ESPs were impregnated into castor leaf discs $(8.5 \mu \mathrm{g} /$ per leaf disc) which were then fed to Sp.littoralis neonates. C is the control with PBS $(n=75)$. 
Changes to the research plan. The main goal of the original research plan was to identify and characterize the insecticidal proteins released by the insect-parasitic nematode Steinernema feltiae and to determine the feasibility of using these proteins as feed bio-toxins against herbivorous pests from two different groups of insects; beetles and larval moths and butterflies. During the course of the project we decided to also evaluate the potential of the proteins released by St.carpocapsae, a parasitic nematode closely related to St.feltiae. We were having some difficulties in producing adequate amounts of St. feltiae ESPs for fractionation as well as for oral toxicity testing and by also investigating St. carpocapsae ESPs we were able to widen the scope of the study. 
Publications for Project US-5090-18

\begin{tabular}{|cccccc|}
\hline $\begin{array}{c}\text { Stat } \\
\text { us }\end{array}$ & Type & Authors & Title & Journal & $\begin{array}{c}\text { Vol:pg } \\
\text { Year }\end{array}$ \\
\hline
\end{tabular}




\section{Appendix (Unpublished data)}

\section{Sc toxins bioassay for $S$. littoralis by leaf disc assay method with concentrations of $8.5 \mu \mathrm{g} / \mathrm{per}$}

\section{leaf disc}

Toxins: Steinernema carpocapsae $(\mathrm{Sc})$ stock concentration $0.6 \mu \mathrm{g} / \mu 1$

Larvae: Spodoptera littoralis

Methodology: Five castor leaf discs $\left(0.28 \mathrm{~cm}^{2}\right.$ leaf area) were impregnated with Sc toxins concentration of $8.5 \mu \mathrm{g} /$ per leaf disc $(14.7 \mu 1$ of Sc toxin was used per leaf disc and its stock concentration is $0.6 \mu \mathrm{g} / \mu \mathrm{l}$ ) to each leaf disc five neonate larvae of $S$. littoralis were added (5 neonates inoculated) and mortality were checked in 24 hours intervals up to $100 \%$ mortality in toxin treated samples. For control in place toxins 1X PBS buffer was used. Experiments were repeated in 3 times. Three repeats were used to calculate percentage of survival and LT50 by using the JMP 14 software (www.jmp.com).

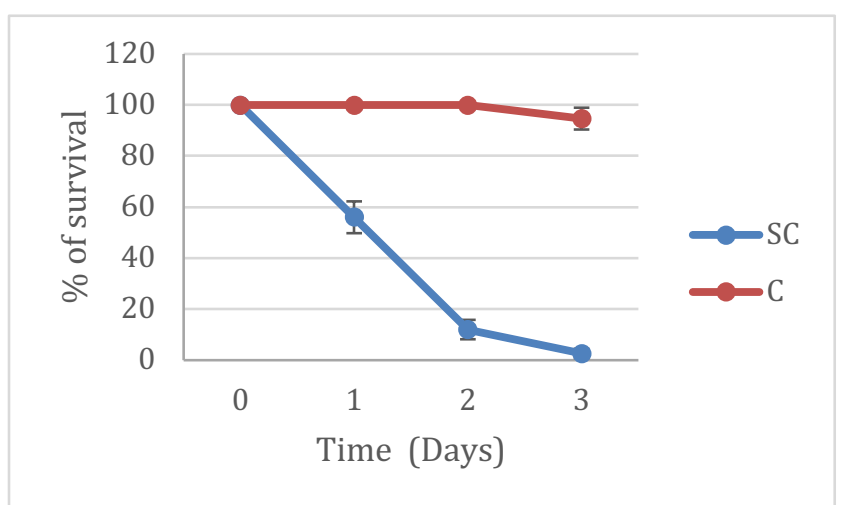

Results: Around 50\% larvae shown paralyzed after 10-15 hours of post inoculation (Not shown in the graph) and mortality started around $24 \mathrm{~h}$ of post inoculation (Fig. 3). After $48 \mathrm{~h}$ (2days) 80 to $100 \%$ mortality were observed. Controls were shown no mortality up to $48 \mathrm{~h}$ later slight mortality were observed.

Figure 3. Sp. littoralis oral toxicity feeding assay. St. carpocapsae ESPs were impregnated into castor leaf discs $(8.5 \mu \mathrm{g} / \mathrm{per}$ leaf disc) which were then fed to Sp. littoralis neonates. C is the control with PBS $(n=75)$.

Lesser feeding on Sc treated leaf discs were observed in comparison to control and shown in the (Fig. 4) of 2 days after the post inoculation. Sc toxins showing LT50 on 1.2 days (Table1).

Fig 1. Survival graph of $S$. littoralis neonates against Sc toxins $(8.5 \mu \mathrm{g} /$ per leaf disc).

Sc is Steinernema carpocapsae toxin, C is Control with $1 \mathrm{X}$ PBS. $(\mathrm{n}=75)$.

Table 1. Median Lethal Time in Days (LT50) of the Sc toxins to the S.littorallis leaf disc impregnation method.

\begin{tabular}{ccccccc}
\hline Sample & LT50(Days) & Std Error & Lower 95\% & Upper 95\% & prob > ChiSq & R square \\
\hline Control & 3.16 & 0.11 & 2.84 & 3.47 & 0.0003 & 0.973483 \\
St. carpocapsae(SC) & 1.20 & 0.11 & 0.89 & 1.52 & & \\
\hline
\end{tabular}




\section{Control on day 2}

C1

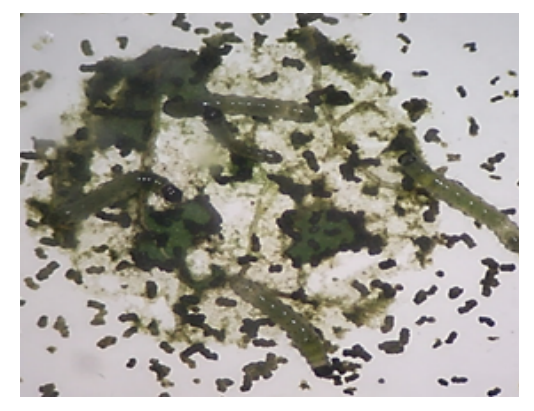

C2

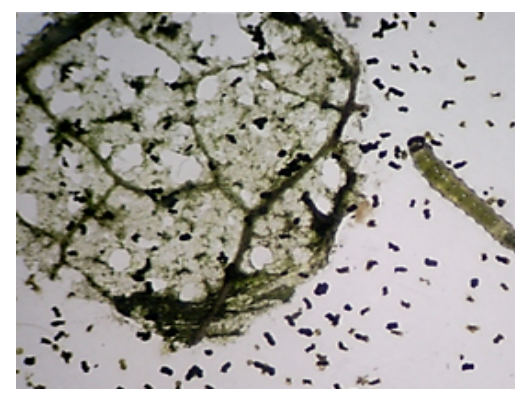

C3

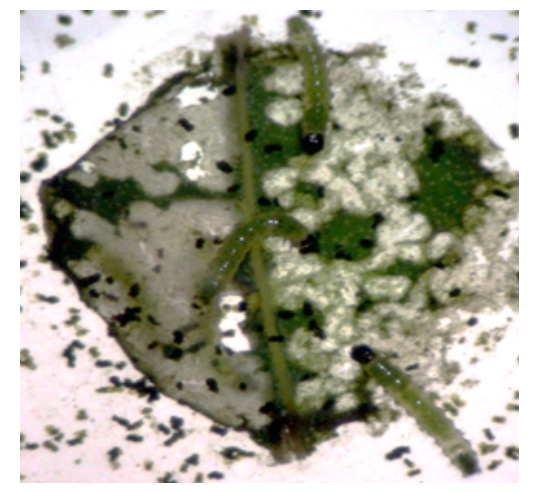

C4

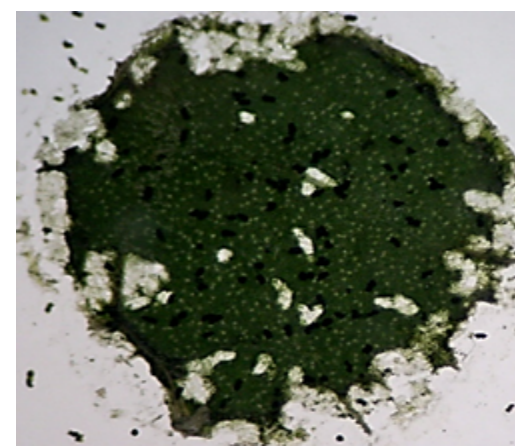

Sc toxins on day 2

SC1

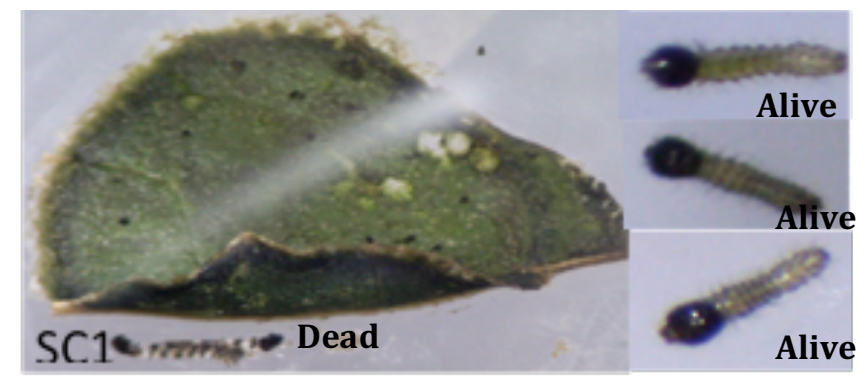

SC2
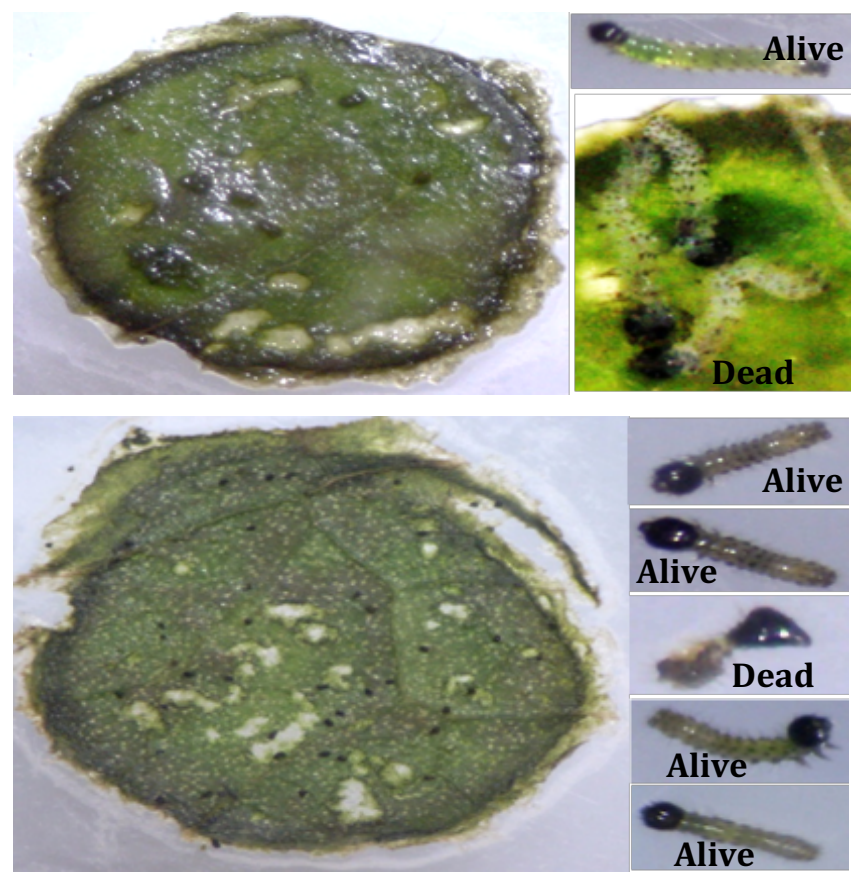

SC4

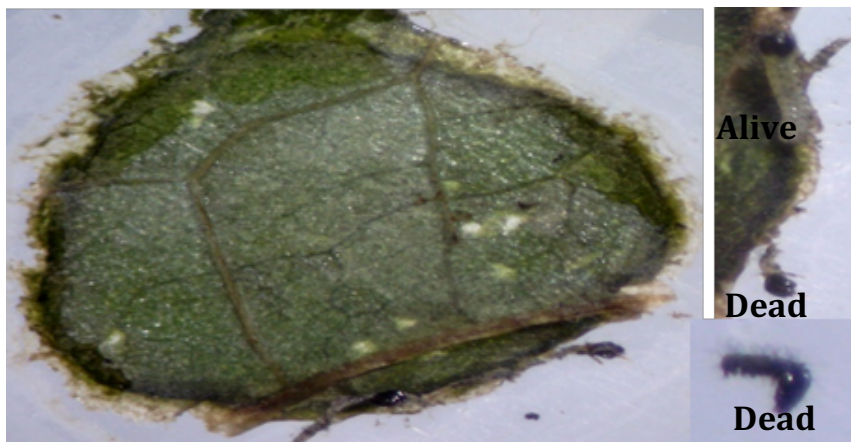


C5

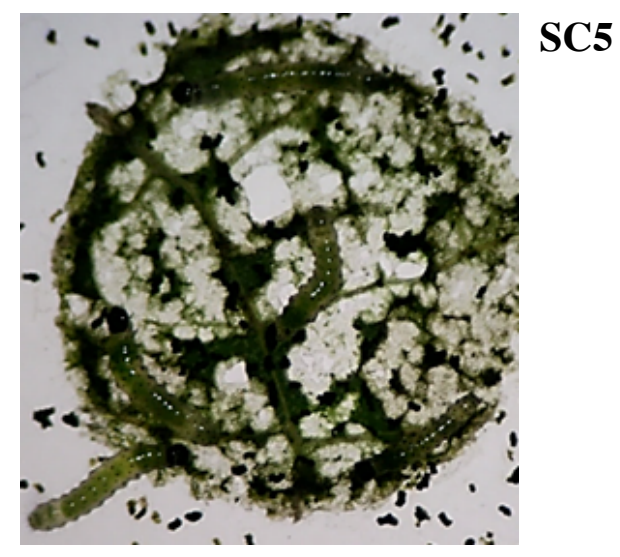

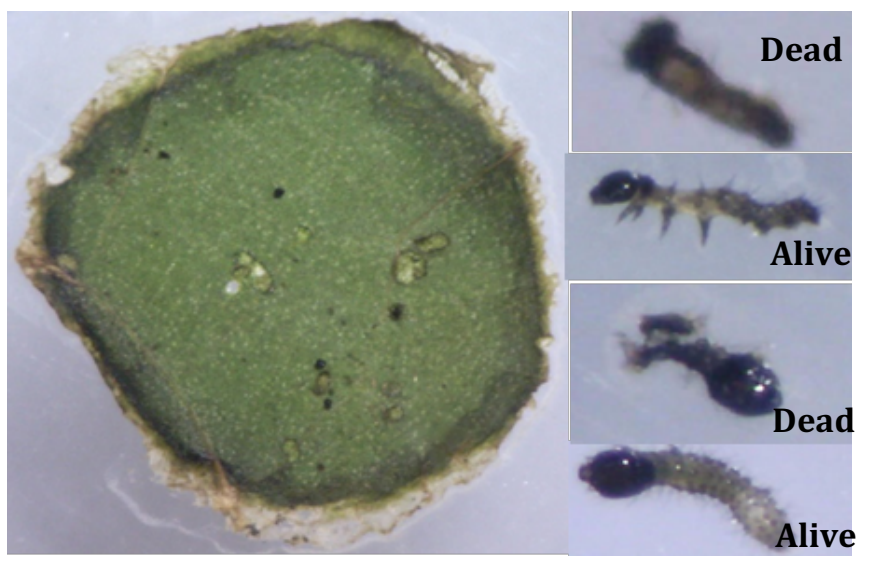

Alive

Figure 4. Showing the feeding effect of $S$. littoralis neonates against Sc toxins $(8.5 \mu \mathrm{g} /$ per leaf disc) treated Caster leaf discs after 2days of post inoculation. SC1 to SC5 is Steinernema carpocapsae toxins treated 5 replicates, C1 to C5 is Controls with 1X PBS 5 replicates. Scale: photographs are in 25x taken in Binocular microscope with Dino-eye camera. (Individual larvae in treated samples are showing the $30 \mathrm{X}$ to $40 \mathrm{X}$ magnifications)

\section{Sc toxins checked for LD50 against Sp. littoralis}

Toxins: Steinernema carpocapsae (Sc) stock concentration $0.6 \mu \mathrm{g} / \mu 1$

Concentrations: $8.5,6.5,4.5,2.5 \mu \mathrm{g} /$ per leaf disc

\section{Larvae: Spodoptera littoralis}

Methodology: Sc toxins concentrations of 8.5, 6.5, 4.5, $2.5 \mu \mathrm{g} /$ per leaf disc were used and each concentration treatment consist of five castor leaf discs $\left(0.28 \mathrm{~cm}^{2}\right.$ leaf area) were impregnated with Sc toxins to each leaf disc five neonate larvae of $S$. littoralis were added (5 neonates inoculated) and mortality were checked in a 24 hours intervals up to $100 \%$ mortality in toxin treated samples. For control in place toxins $1 \mathrm{X}$ PBS buffer was used.

Results: LD50 concentrations at day1 shows $0.65 \mu \mathrm{g}$, Day2 shows $5.8 \mu \mathrm{g}$, Day3 shows $4.09 \mu \mathrm{g}$, day4 $2.28 \mu \mathrm{g}$, day5 shows $63 \mu \mathrm{g}$ per leaf disc (Table 2).

Table 2. Median Lethal Dose (LD50) of the Sc toxins to the S.littorallis leaf disc impregnation method

\begin{tabular}{ccccc}
\hline Time (Days) & $\mathrm{n}$ & LD 50 & Lower 95\% & Upper 95\% \\
\hline 1 & 25 & 8.975887 & 7.26068 & 13.51257 \\
2 & 25 & 5.801526 & 4.69824 & 7.30251 \\
\hline
\end{tabular}




\begin{tabular}{llccc}
\hline 3 & 25 & 4.090367 & 2.85296 & 5.26126 \\
4 & 25 & 2.27792 & 0.41798 & 3.46429 \\
5 & 25 & 0.629013 & -2.4337 & 2.07773 \\
\hline
\end{tabular}

\section{Checking of SC toxins to Beetles artificial diet method}

Toxins: Steinernema carpocapsae (Sc) $5 \mu \mathrm{g} / \mathrm{gm}$ of diet

Larvae: Alphitobius diaperinus (Lesser meal worm beetle)

Methodology: $2.15 \mathrm{~g}$ of diet was taken it consists $0.56 \mathrm{~g}$ of Heliothis premix and $1.5 \mathrm{ml}$ of $1 \mathrm{X}$ PBS in addition to this toxins were added $5 \mu \mathrm{g} / \mathrm{g}$ of diet. To the $2.15 \mathrm{~g}$ of diet Sc $6.32 \mu 1$ from Stock of $1.7 \mu \mathrm{g} / \mu 1$ (equal to $10.75 \mu \mathrm{g}$ ) Sf $15.579 \mu 1$ from stock of $0.690 \mu \mathrm{g} / \mu 1$ (equal to $10.75 \mu \mathrm{g}$ ) added. Diet made in to 5 bigger pellets and 5 neonate larvae were added in each plate and checked for the mortality.

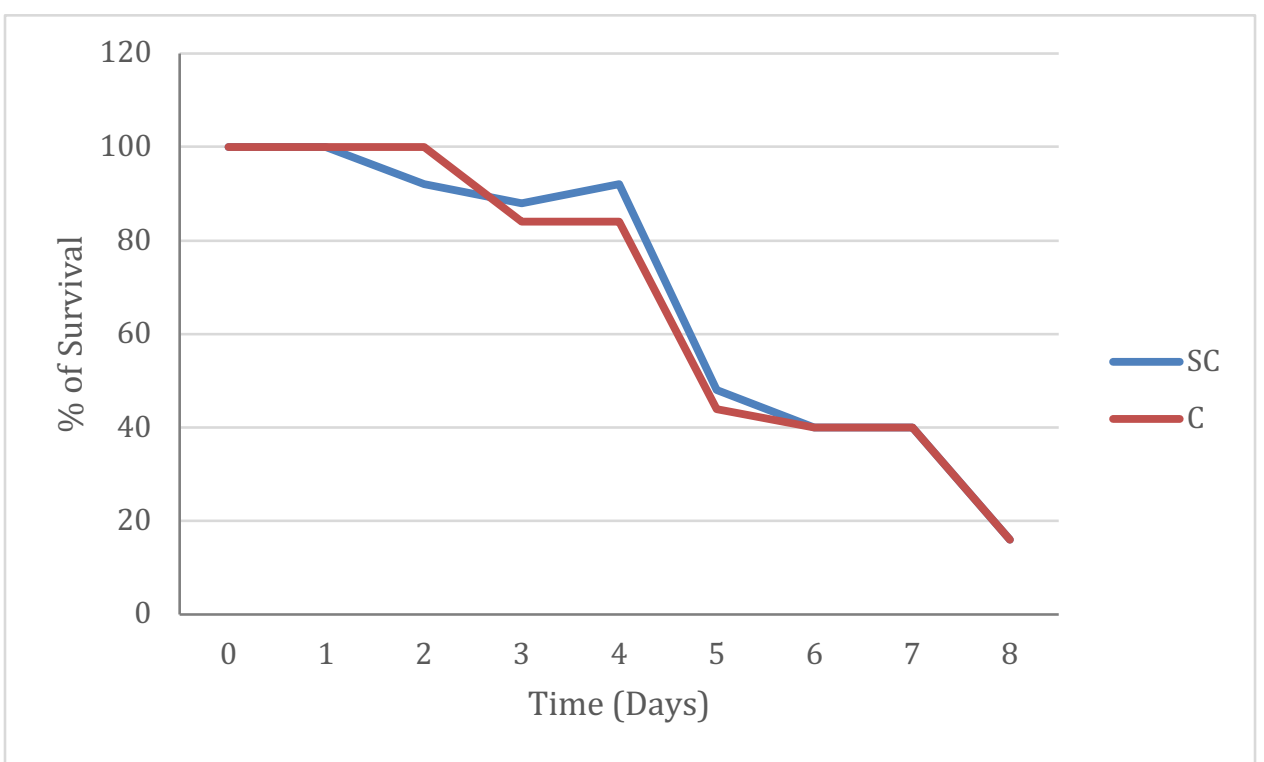

No difference were shown between sc toxins and control.

\section{Checking of SC toxins to Beetles droplet method}

Toxins: Steinernema carpocapsae (Sc) stock concentration $0.6 \mu \mathrm{g} / \mu 1$

Larvae: Alphitobius diaperinus (Lesser meal worm beetle)

Methodology: For the each treatment has five petriplates $(55 \mathrm{~mm})$ and each petriplate added Sc and Sf toxins equalent to $8.58 .5 \mu \mathrm{g}$ and ten larvae 1 st to $2^{\text {nd }}$ instar lesser mealworm larvae were added and mortality were checked in a 24 hours intervals up to $100 \%$ mortality in toxin treated 
samples. For control in place toxins 1X PBS buffer was used. Experiments were repeated in 2 times. Two repeats were used to calculate percentage of survival and LT50 by using the JMP 14 software.

Results: Sc toxins were checked to Alphitobius diaperinus by droplet method after 48 hours of treatment mortalies were observed. LT 50 of Sc toxins to Alphitobius diaperinus is 4.9days.

\begin{tabular}{ccccccc}
\hline \multirow{2}{*}{ Level } & & & Std & Lower & Upper & \\
& $\mathrm{n}$ & LT50(Days) & Error & $95 \%$ & $95 \%$ & \multirow{2}{*}{ R square } \\
\hline Control & 100 & 27.4962 & 1.9836 & 18.96 & 36.031 & 0.969995
\end{tabular}

Steinernema carpocapsae

(SC)

$100 \quad 4.9397 \quad 1.9836 \quad-3.6$

13.474

\section{Checking the Sc nematodes to the Sp. littoralis}

EPN: Steinernema carpocapsae $(\mathrm{Sc})$ nematodes

Concentrations: $1 \mathrm{million} / \mathrm{cm}^{2}\left(55 \mathrm{~mm}\right.$ petriplate $2.3 \mathrm{~cm}^{2}$ area, around 2350 nematodes $)$

Larvae: Spodoptera littoralis

Methodology: Five $55 \mathrm{~mm}$ petriplates arranged the whatmann paper added the $2350 \mathrm{Sc}$ nematodes in a $1 \mathrm{ml}$ volume and castor leaf discs, ten neonate larvae of $S$. littoralis were added and mortality were checked in 24 hours intervals up to $100 \%$ mortality in Sc treated samples. For control in place toxins $1 \mathrm{ml}$ of distilled water was used.

Results: Mortalies were observed 48hours of post inoculation. LT 50 of Sc nematodes is 2.8days.

\begin{tabular}{ccccc}
\hline & & & Lower \\
Sample & $\mathrm{n}$ & LT50 (Days) & $95 \%$ & Upper 95\% \\
\hline $\begin{array}{c}\text { Control } \\
\text { Steinernema carpocapsae }\end{array}$ & 50 & 10.35218 & -2764.31 & 2785.491 \\
$(\mathrm{SC})$ & 50 & 2.80584 & 2.61 & 3 \\
\hline
\end{tabular}

$\mathrm{n}=$ sample size, LT50: Lethal time for the fifty percent mortality

\section{SF toxins checking on Sp. littoralis 5 and $8.5 \mu \mathrm{g} /$ per leaf disc}

Toxins: Steinernema feltiae (Sf)

Larvae: Spodoptera littoralis 
Methodology: For each concentration five castor leaf discs $\left(0.28 \mathrm{~cm}^{2}\right.$ leaf area) were impregnated with Sf toxins concentration of 5 and $8.5 \mu \mathrm{g} /$ per leaf disc to each leaf disc five neonate larvae of S. littoralis were added (5 neonates inoculated) and mortality were checked in a 24 hours intervals up to $100 \%$ mortality in toxin treated samples. For control in place toxins 1 X PBS buffer was used.

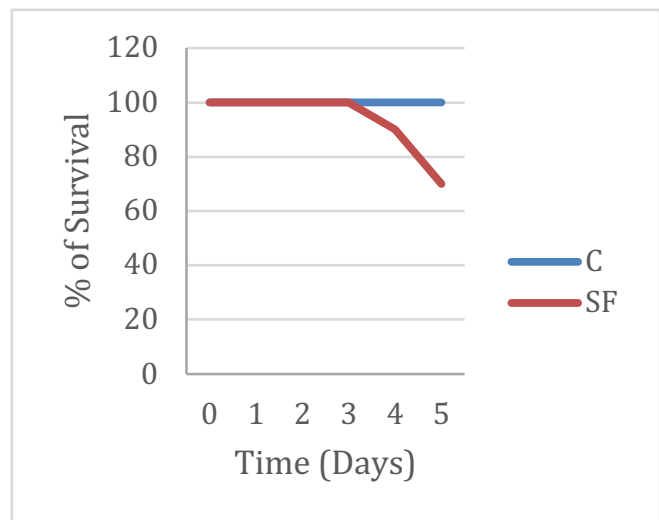

$8.5 \mu \mathrm{g} /$ per leaf disc

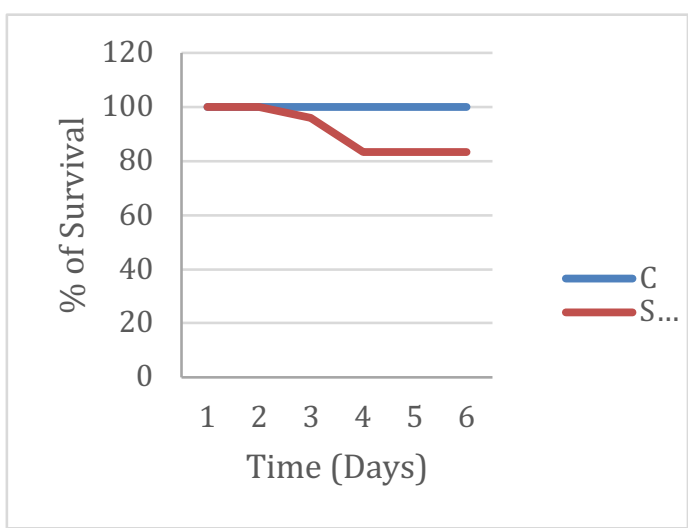

$5 \mu \mathrm{g} /$ per leaf disc

Results: Sf toxins 5 and $8.5 \mu \mathrm{g} /$ per leaf disc concentrations were used. Mortalies were observed after the 2-3days of post inoculation.

\section{Checking the Sf nematodes to the $S$. littoralis}

EPN: Steinernema feltiae (Sf) nematodes

Concentrations: $1 \mathrm{million} / \mathrm{cm}^{2}\left(55 \mathrm{~mm}\right.$ petriplate $2.3 \mathrm{~cm}^{2}$ area, around 2350 nemarodes)

Larvae: Spodoptera littoralis

Methodology: Five $55 \mathrm{~mm}$ petriplates arranged the whatmann paper added the $2350 \mathrm{Sf}$ nematodes in a $1 \mathrm{ml}$ volume and castor leaf discs, ten neonate larvae of $S$. littoralis were added and mortality were checked in a 24 hours intervals up to $100 \%$ mortality in Sc treated samples. For control in place toxins $1 \mathrm{ml}$ of distilled water was used.

Results: Mortalies were observed 48hours of post inoculation.LT 50 of Sf nematodes is 4.25 days.

\begin{tabular}{ccccc}
\hline & & \multicolumn{3}{c}{ Lower } \\
Sample & $\mathrm{n}$ & LT 50 & $95 \%$ & Upper 95\% \\
\hline Control & 50 & 17.0394 & -3260.84 & 3296.53 \\
Steinernema feltiae (SF) & 50 & 4.25 & $3.87 \mathrm{E}+00$ & $4.76 \mathrm{E}+00$ \\
\hline
\end{tabular}

$\mathrm{n}=$ sample size, LT50: Lethal time for the fifty percent mortality 


\section{References}

Lu, D., Macchietto, M., Chang, D., Barros, M.M., Baldwin, J., Mortazavi, A., and Dillman, A.R. (2017). Activated entomopathogenic nematode infective juveniles release lethal venom proteins. PLoS Pathog 13, e1006302. 